\title{
Le rôle de l'immersion dans l'apprentissage du schwa chez les apprenants alémaniques avancés de FLE
}

\author{
Romain Isely ${ }^{1, *}$, Isabelle Racine ${ }^{1}$, Sylvain Detey ${ }^{2}$, Helene N. Andreassen ${ }^{3}$, et Julien \\ Eychenne ${ }^{4}$ \\ ${ }^{1}$ ELCF, Université de Genève, 5, rue de Candolle, 1211 Genève 4, Suisse \\ ${ }^{2}$ Waseda University, 1-6-1 Nishi-Waseda, Shinjuku-ku, 169-8050 Tokyo, Japon \\ ${ }^{3}$ Université Arctique de Norvège, Breivika, 9037 Tromsø, Norvège \\ ${ }^{4}$ Hankuk University of Foreign Studies, Mohyeon, Yongin, 17035 Gyeonggi, République de Corée
}

\begin{abstract}
Résumé. Dans l'apprentissage du français, le schwa constitue un véritable défi pour les apprenants. Dans ce travail, effectué dans le cadre du projet «Interphonologie du Français Contemporain » (IPFC), nous proposons un système de codage alphanumérique des réalisations du schwa d'apprenants de FLE et en illustrons la pertinence à l'aide de données d'apprenants suisses alémaniques avancés, issues de deux des tâches du protocole IPFC (lecture d'un texte et conversation guidée). Les résultats montrent que, si un séjour de longue durée en milieu francophone ne semble pas avoir d'effet à lui seul sur le comportement du schwa, il interagit néanmoins avec la tâche - plus de chute du schwa après un séjour dans les conversations mais pas en lecture - ainsi qu'avec le type de mots - après un séjour, le schwa chute davantage dans les monosyllabes mais n'a pas d'effet sur les polysyllabes, que le schwa soit en syllabe initiale ou interne. Le séjour semble ainsi aider les apprenants à acquérir une compétence sociolinguistique se rapprochant de celle des natifs. Ces résultats préliminaires ouvrent des pistes didactiques afin de fournir du matériel pédagogique permettant de ne pas laisser l'appropriation de cette compétence à la portée des seuls apprenants pouvant effectuer un séjour en immersion mais de la travailler également de manière efficace en classe.
\end{abstract}

\begin{abstract}
The effect of studying abroad on the acquisition of schwa in advanced Swiss German learners of French. Mastering 'schwa' is one of the major challenges that non-native speakers face when learning French as a foreign language. The present study, set within the framework of the IPFC project ("Interphonologie du Français Contemporain"), describes the schwa-coding system applied to the IPFC corpus data and is illustrated here with data from Alemannic-speaking Swiss learners of French (two tasks: text reading and guided conversation). Our results show that, even though studying abroad in a French-speaking area alone does not have a direct significant impact on schwa's behavior, it does interact with two variables: the type of task (more schwa deletion after studying abroad
\end{abstract}

\footnotetext{
*Corresponding author : Romain.Isely@unige.ch.
} 
observed in the conversation but not in the reading task) and the type of word (more schwa deletion after studying abroad observed in monosyllabic words but not in polysyllabic words, both in word-initial or word-medial syllabic positions). In this respect, studying abroad seems to help learners achieve native-like sociolinguistic proficiency. These first results pave the way for the development of in-class pedagogical material which could help learners achieve such a level of proficiency, whether or not they have the opportunity to study abroad in a French-speaking area.

\section{Introduction}

Comprendre et maîtriser le schwa, voyelle qui, en français, a la particularité de pouvoir ou non être réalisée (ex. "petit» peut être prononcé [pəti] ou [pti]), représente généralement un véritable défi pour les apprenants de français langue étrangère (ci-après FLE), que ce soit sur le plan de la perception (Stridfeldt, 2005, Nouveau, 2012) ou de la production (Thomas, 2001, Uritescu et al., 2002 et 2004). Comme le relèvent Nouveau et Detey (2007), les apprenants doivent en effet être capables de comprendre qu'une même séquence, représentée par une seule forme écrite, peut correspondre à une multiplicité de

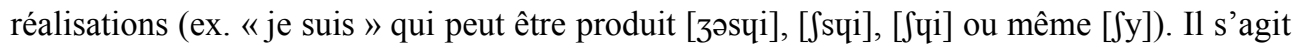
là d'une compétence très rapidement nécessaire dans l'apprentissage de l'oral, puisque, sans elle, la compréhension de la langue s'en voit grandement affectée. Sur le plan de la production, outre la difficulté généralement représentée par le timbre du schwa - souvent produit $[\varepsilon]$ ou $[\mathrm{e}]$ (pour une synthèse, voir Detey et al., 2016a) -, les apprenants doivent savoir, d'une part, que certaines formes peuvent alterner (p. ex. "semaine», [səmen]/[smen]), alors que d'autres non (p. ex. « casserole » est habituellement produit sans schwa, [kassol] $]^{1}$, tandis que, dans «vendredi », le schwa se maintient, [vãdsədi]) et, d'autre part, produire la forme attendue dans un contexte donné. Si la production systématique de formes avec schwa peut constituer une bonne stratégie d'évitement dans un premier temps, la maîtrise de l'alternance des formes avec et sans schwa permet d'améliorer sensiblement la compétence sociolinguistique des apprenants lorsqu'ils ont atteint un niveau intermédiaire/avancé. Or, comme l'ont montré différentes études (voir, entre autres, Howard et al., 2013, Mougeon et al., 2002, Regan et al., 2009), ce type de compétence s'avère tout aussi important que l'acquisition du système lui-même.

Ainsi, les enjeux concernant le schwa sont vastes et diffèrent fortement suivant le moment de l'apprentissage, ce qui explique sans doute pourquoi les manuels de français spécialisés en prononciation consacrent généralement toujours un chapitre à cette question. Dans leur présentation du phénomène, ces manuels (voir entre autres Abry \& Chalaron, 2011, Charliac \& Motron, 2014, Martins \& Mabilat, 2004) font référence presque systématiquement à la «loi des trois consonnes » établie par Grammont (1914), qu'ils nuancent en fonction de la position du schwa dans le mot. Si la majorité des règles présentées se situent donc essentiellement à un niveau segmental, d'autres variables, d'ordre plutôt sociolinguistique (p. ex. la variation diatopique, le style de discours, le registre ou encore le débit), sont parfois mentionnées, mais elles se limitent généralement à une simple énumération. Le traitement proposé, confiné majoritairement à des considérations segmentales et positionnelles, ne semble cependant pas suffisant pour permettre le développement d'une compétence sociolinguistique satisfaisante. Comme le souligne Thomas (2001), on peut par conséquent se demander par quels moyens les apprenants peuvent acquérir ce type de compétence, alors qu'ils ne bénéficient pas d'un contact constant avec la langue orale et que, comme le relèvent Paternostro et al. (2017), 
cette compétence n'est que rarement didactisée en classe de FLE. Il peut donc en résulter de sérieuses difficultés à communiquer de manière adéquate en situation " réelle ».

Pour répondre à cette question, il semble nécessaire d'observer comment les apprenants gèrent le schwa en production. Outre des difficultés liées au timbre du schwa, notamment en raison de l'influence de la L1 (Andreassen \& Lyche, 2016, Boubnova \& Ratnikova, 2016, Stridfeldt, 2005), deux considérations majeures ressortent des travaux qui se sont penchés sur ce sujet avec différentes populations d'apprenants : a) les deux facteurs ciblés dans les manuels de prononciation, à savoir l'environnement segmental et la position du schwa dans le mot, jouent un rôle important sur son maintien et sa chute, mais de manière assez similaire entre les apprenants et les natifs (Andreassen \& Lyche, 2016, Boubnova \& Ratnikova, 2016, Nouveau \& Detey, 2007, Thomas, 2001, Uritescu et al., 2002 et 2004); b) si les apprenants ont tendance à davantage maintenir le schwa que les natifs, leur taux de chute du schwa augmente avec l'immersion et le contact avec des natifs, leur permettant ainsi de se rapprocher des usages de ces derniers (Thomas, 2001, Uritescu et al. 2002 et 2004). Katanaeva-Morozova (2008), dans une étude pilote, a tenté de creuser cette question des usages en cherchant à déterminer l'«expérience » que des apprenants russophones avancés, en situation d'immersion, ont de ceux-ci, à travers l'obtention d'indices de fréquence estimée des formes de mots avec et sans schwa, indices qu'elle a comparés à ceux obtenus par Racine (2008) avec des natifs. Elle observe que les apprenants se basent essentiellement sur les aspects positionnels et segmentaux pour décider du maintien/chute du schwa, car ils ne disposent pas de la même « expérience » de l'usage des formes avec et sans schwa que les natifs.

L'ensemble de ces travaux semble ainsi refléter l'apprentissage proposé par les manuels de phonétique. Or, si de nombreux travaux ont tenté d'expliquer le comportement du schwa en français L1 (pour une synthèse, voir Racine, 2008), il en ressort clairement que les aspects positionnels et segmentaux ne suffisent pas à en rendre compte, mais que de nombreux facteurs interagissent de manière complexe. L'apport de la linguistique de corpus (Durand, 2009) à ce domaine semble donc intéressant puisque susceptible d'offrir un cadre adéquat à une prise en compte large du contexte, par le biais de l'examen de productions issues de grands corpus oraux, que ce soit en L1 ou en L2. Or, si les corpus oraux en français L1 ont été l'objet d'un important développement ces dernières années, la même démarche n'a débuté que plus récemment en L2, et ce, pour le français, entre autres, à travers le projet "Interphonologie du Français Contemporain » (dorénavant «IPFC », Detey \& Kawaguchi, 2008, Racine et al., 2012, Detey et al., 2016b, Detey \& Racine, 2017), cadre dans lequel se situe le travail présenté ici.

Dans ce qui suit, nous commençons par présenter brièvement le projet IPFC avant d'exposer le traitement du schwa qui y est proposé, par le biais d'un codage alphanumérique des réalisations des apprenants. Nous illustrons ensuite les possibilités d'analyse qu'offre ce type de traitement en présentant une première étude des réalisations du schwa et des facteurs qui en conditionnent le maintien ou la chute chez des apprenants alémaniques de FLE de niveau avancé.

\section{Le traitement du schwa dans le projet IPFC}

\subsection{Le projet IPFC}

Le projet IPFC constitue le volet non natif du programme PFC (Durand et al., 2009) et il en adopte, à ce titre, les grandes lignes directrices, à savoir une récolte de données via un protocole identique, constitué de six tâches (répétition d'une liste spécifique de mots, lecture de la liste de mots PFC, lecture de la liste spécifique de mots, lecture d'un texte, entretien guidé avec un natif et conversation libre entre deux apprenants), ce qui permet une 
comparabilité des données à la fois avec des natifs et entre apprenants de différentes L1. Le protocole fournit environ une heure de données par participant et est complété par un questionnaire sociolinguistique visant à établir le profil de l'apprenant.

Sur le modèle PFC, les données sont alignées sur le signal sous Praat (Boersma \& Weenink, 2015) et transcrites orthographiquement selon les conventions adaptées aux spécificités de la parole en L2 (Racine et al., 2011). Si, comme le relève Delais-Roussarie (2009) pour la parole native, la transcription orthographique est déjà en soi une forme d'interprétation, cette question se pose de manière encore plus accrue lorsque l'on s'intéresse aux productions d'apprenants (Mac Whinney, 2015). Il est alors aisé de comprendre pourquoi une transcription complète des productions en API (Alphabet Phonétique International) - ou tout autre système de transcription phonétique -, n'est pas réaliste. Une transcription phonémique consisterait en effet, comme le soulignent Durand \& Lyche (2003), à «mettre la charrue avant les bœufs» puisqu'elle impliquerait irrémédiablement que le système que l'on cherche à établir par le biais de l'enquête a déjà été découvert. Une transcription de type allophonique se heurterait quant à elle à la question du degré de finesse phonétique que l'on cherche à atteindre, "bon nombre de réalisations ne correspondant pas à des choix binaires mais à des valeurs sur des échelles continues » (Durand \& Lyche, 2003 : 230). Comme cela a été fait dans PFC pour la liaison et le schwa, l'approche par le biais d'un codage alphanumérique a donc également été privilégiée dans le cadre du projet IPFC (Detey, 2012 et 2014).

Ce codage permet d'inclure à la fois des champs descriptifs, tels que par exemple la position de l'élément ciblé dans le mot ou le contexte segmental précédent ou suivant, et des champs pour lesquels l'évaluation perceptive de l'élément ciblé doit être effectuée, tels que la présence/absence de l'élément ou l'adéquation de son timbre. Cette analyse privilégie donc une approche perceptive, qui, selon Derwing \& Munro (2015), est indispensable lorsqu'il s'agit de parole non native, et constitue donc, à ce titre, l'une des originalités du projet. Des analyses acoustiques peuvent être menées, dans un deuxième temps, par exemple sur les formes jugées déviantes, de manière à établir les corrélats acoustiques qui ont conduit à cette analyse perceptive. La validité de cette approche par codage a été évaluée, pour les voyelles nasales et arrondies (Detey et al., 2014, Racine, 2012, Racine \& Detey, sous presse), en comparant les résultats de tests de perception classiques effectués par des locuteurs natifs non experts à ceux obtenus sur la base du codage. Les réalisations des apprenants sont donc étiquetées grâce à un codage alphanumérique, établi pour un phénomène donné - à ce stade, un codage spécifique a été développé pour les voyelles orales, nasales, les consonnes et groupes consonantiques, la liaison et pour certains aspects prosodiques. La procédure de codage est généralement effectuée par un codeur natif et est en principe vérifiée par un deuxième évaluateur, pour autant qu'une procédure de double-codage en aveugle ne soit pas nécessaire. Le concordancier Dolmen (Eychenne \& Paternostro, 2016) permet de comparer aisément les codages effectués par les différents codeurs et de calculer leur degré de corrélation (ICC : intraclass correlation coefficient) pour l'ensemble du codage ou pour chaque champ séparément. Dolmen interagissant avec Praat, les erreurs concernant les champs descriptifs peuvent ainsi être corrigées facilement, directement dans les fichiers TextGrid. En revanche, en ce qui concerne les divergences éventuelles dans les champs concernant l'évaluation perceptive, qui ne peuvent évidemment pas être considérées comme des erreurs, une procédure spécifique doit être mise en place ( $\mathrm{p}$. ex. recours à un troisième évaluateur).

Ce codage permet ainsi d'obtenir rapidement et facilement des informations phonéticophonologiques détaillées sur divers phénomènes de l'oral. Une interface Dolmen spécifique à chaque codage IPFC permet par la suite d'effectuer des requêtes ciblées dans les données codées afin d'en élaborer une description fine. 


\subsection{Le codage schwa}

Afin de développer un codage pour le schwa, qui, dans la ligne définie par Laks (2015) pour la liaison, vise à décrire sans «a priori » les réalisations des apprenants, nous avons pris le parti de considérer comme un schwa toute unité phonético-phonologique correspondant à un $<\mathrm{e}>$ graphique, à l'exception de ceux se trouvant en syllabe fermée (ex. «mer» $[\mathrm{m \varepsilon s}]$ ). Nous avons en effet dû considérer le fait que la notion de schwa ne recouvre pas obligatoirement la même réalité pour les apprenants que pour les natifs. Ainsi, dans PFC, la voyelle précédée d'une suite constituée d'une obstruante suivie d'une liquide (ex. " premier », « vendrédi ») n'est pas codée puisqu'elle ne chute jamais dans ce contexte. Un apprenant ne pouvant a priori pas le savoir, ces contextes sont donc codés dans IPFC. Nous avons en revanche décidé de ne pas coder les $<\mathrm{e}\rangle$ graphiques finaux (ex. " arbre $»)$, qui sont codés dans PFC puisque les usages méridionaux y font l'objet d'une attention toute particulière. En effet, l'alternance dans ce type de réalisations chez les apprenants, même si elle peut se révéler intéressante, ne nous semblait pas primordiale pour l'apprentissage de la compétence sociolinguistique. La procédure de codage étant extrêmement chronophage, nous avons préféré nous concentrer sur des réalisations plus susceptibles de varier, telles que le schwa des monosyllabes (ex. «le $»$, « je $»)$, de syllabe initiale (ex. "semaine », " premier ») et interne de polysyllabes (ex. « samedi », " gouvernement »).

Inspiré par le code PFC, le système mis en place dans IPFC a dû être adapté de manière à prendre en compte certaines spécificités de la L2 (p. ex. le timbre avec lequel le schwa est réalisé). Le format a également été revu, de manière à conserver une certaine cohérence avec les autres codes du projet. Le code IPFC pour le schwa comprend donc six champs, séparés par un tiret bas. Les quatre premiers champs portent sur des éléments descriptifs, alors que les deux derniers ciblent l'évaluation perceptive du schwa. Il se présente ainsi :

- Champ 1 : nature du phénomène ciblé par le codage ;

- Champ 2 : position syllabique du schwa dans le mot;

- Champ 3 : contexte segmental situé immédiatement à gauche du schwa ;

- Champ 4 : contexte segmental situé immédiatement à droite du schwa ;

- Champ 5 : présence/absence du schwa;

- Champ 6 : timbre du schwa, s'il est réalisé.

Le champ 1 permet simplement d'indiquer quel phénomène linguistique est ciblé dans le codage. Le code correspondant à celui du schwa est 08 . Le champ 2 indique quant à lui si le schwa se trouve dans un monosyllabe - indiqué par le code $1-$, dans la syllabe initiale d'un polysyllabe - indiqué par le code 2 - ou dans la $2^{\mathrm{e}}, 3^{\mathrm{e}}, 4^{\mathrm{e}}$, etc. syllabe (non finale) d'un polysyllabe - indiqué par le code 3 .

Le champ 3 permet de décrire le contexte précédant immédiatement le schwa. On distingue quatre contextes possibles : a) le schwa est précédé d'une seule consonne qui, elle-même, se trouve en début de groupe intonatif. Dans ce cas, on indique le chiffre 1 suivi de la nature de la consonne qui précède le schwa $(\mathrm{P}=$ plosive, $\mathrm{N}=$ nasale, $\mathrm{F}=$ fricative, $\mathrm{L}=$ liquide, $\mathrm{S}=$ semi-voyelle) suivi de 00 . Ainsi, p. ex. «Levez-vous » sera codé $1 \mathrm{~L} 00$; b) le schwa est précédé d'une seule consonne qui, elle-même, est précédée d'une pause ou d'une hésitation. Dans ce cas, on indique le chiffre 2, suivi de la nature de la consonne qui précède le schwa $(\mathrm{P} / \mathrm{N} / \mathrm{F} / \mathrm{L} / \mathrm{S})$, suivi de 00 . Ainsi, p. ex. " un euh requin » sera codé $2 \mathrm{~L} 00$; c) le schwa est précédé d'une consonne et d'une voyelle $(\mathrm{V}(\#) \mathrm{Cə})^{2}$. Ce contexte sera codé $3 \mathrm{~V}$, suivi de 0 ou de 1 et de la nature de la consonne, 0 indiquant que la voyelle précédente est également un schwa, 1 étant utilisé pour toute autre voyelle. Ainsi, " le petit » sera codé $3 \mathrm{~V} 0 \mathrm{P}$, alors que « un petit » sera codé $3 \mathrm{~V} 1 \mathrm{P}$ ) ; d) le schwa est précédé de deux consonnes (ou plus mais seules les deux consonnes précédant immédiatement le schwa sont prises en compte dans le codage), soit une structure de type $\mathrm{C}(\#) \mathrm{C}$. Ce contexte sera codé 4 suivi de 
la nature de la consonne immédiatement à gauche du schwa, de 0,1 ou 2 , et de la nature de la $2^{\text {ème }}$ consonne. Le chiffre 0 indique qu'il n'y a pas de frontière lexicale entre les deux consonnes (ex. " grenier » sera codé $4 \mathrm{P} 0 \mathrm{~L}), 1$ est utilisé dans les cas où il y a une frontière lexicale entre les deux consonnes (ex. "neuf semestres », produit [nœfsəmests], sera codé 4F1F). Le chiffre 2 indique non seulement qu'il y a une frontière entre les deux consonnes mais aussi que le premier mot se termine par un $<\mathrm{e}>$ graphique qui est prononcé (ex. « chaque semestre », produit [Jakəِsəmestr], sera codé 4P2F).

Le champ 4 permet de décrire le contexte suivant immédiatement le schwa. On distingue également quatre contextes possibles : a) le schwa est suivi par une pause ou une hésitation, ce qui sera codé 10, comme p. ex. dans « il a dit de ... manger »; b) le schwa est suivi par une voyelle, ce qui sera codé 20 , comme p. ex. dans « le hasard »; c) le schwa est suivi par une consonne, elle-même suivie d'une voyelle qui n'est pas un schwa, ce qui sera codé 3 suivi de la nature de la consonne (ex. «demain » sera codé $3 \mathrm{~N}$ ) ; d) le schwa est suivi par une consonne, elle-même suivie par un autre schwa, ce qui sera codé 4 suivi de la nature de la consonne (ex. « il se secoue » sera codé 4F).

Si les quatre premiers champs du codage sont purement descriptifs, l'aspect perceptif intervient pour les deux derniers. Le champ 5 indique en effet si le schwa est réalisé ou non. 0 indique que le schwa est absent (ex. « fenêtre » réalisé [fnetь]), 1 indique que le schwa est présent (ex. «fenêtre » réalisé [fəncts]), alors que 2 indique que le schwa est présent mais réalisé avec une hésitation (ex. «fenêtre » produit [fərncts]). Cette dernière indication est nécessaire car, si le locuteur se sert du schwa pour hésiter, celui-ci n'est donc pas susceptible de chuter, même si le contexte s'y prêterait. Le champ 6 permet quant à lui d'indiquer, lorsque le schwa est présent, le timbre utilisé pour sa réalisation. On distingue ici quatre possibilités : a) s'il n'est pas présent (indiqué par 0 dans le champ 5), la question du timbre est non pertinente, ce qui est indiqué par le code $000 ; b)$ s'il est réalisé avec un timbre plutôt fermé, s'apparentant à [ø], on indique 150 ; c) s'il est réalisé avec un timbre plutôt ouvert, s'apparentant à [œ], il est codé avec le chiffre 180 ; d) s'il est réalisé comme une autre voyelle, il sera codé 2 suivi du code de la voyelle perçue (en reprenant ici les codes utilisés dans le champ 1 du système de codage développé pour les voyelles orales et nasales, à savoir $10=[\mathrm{i}], 20=[\mathrm{y}], 30=[\mathrm{u}], 40=[\mathrm{e}], 60=[\mathrm{o}], 70=[\varepsilon], 90=[\mathrm{o}], 11=[\mathrm{a}]$, $12=[\tilde{a}], 13=[\tilde{o}], 14=[\tilde{\varepsilon}]$ et $15=[\tilde{\varrho}])$.

Le codage de l'ensemble des champs se fait sous Praat, dans une tire dédiée, générée à partir de la duplication de la tire contenant la transcription orthographique des productions de l'apprenant. Ainsi, par exemple, comme l'illustre la Figure 1 ci-dessous, la séquence « je parle le suisse allemand », dans laquelle le schwa est réalisé dans "je » mais pas dans «allemand», sera codée ainsi : «je08_1_2F00_3P_1_180 parle (le/0 $)^{3}$ suisse alle08_3_3V1L_3N_0_000mand», la tire spécifique pour le codage schwa, nommée «codschwa », étant celle située juste au-dessous du spectrogramme.

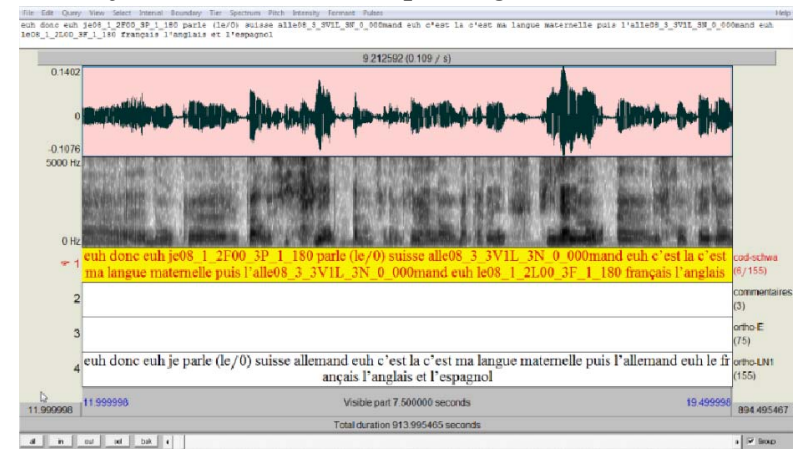


Fig. 1. Exemple de codage du schwa sous Praat dans une conversation guidée d'une apprenante alémanique.

Ainsi, le traitement du schwa par le biais d'un système de codage alphanumérique que nous proposons permet, une fois l'étape de codage réalisée, grâce à l'interface spécifique développée à cet effet dans Dolmen, une analyse relativement fine et détaillée d'une grande quantité de données, en tenant compte de différents paramètres : type d'apprenants, tâche, type de mots, position du schwa, environnement segmental, etc.

\section{Une illustration : le schwa chez les apprenants alémaniques}

Afin d'illustrer les possibilités offertes par le système de codage expliqué précédemment, nous présentons, dans ce qui suit, une étude préliminaire visant à analyser les réalisations de schwa d'apprenants suisses alémaniques de FLE, de niveau avancé, étude qui s'inscrit dans le cadre d'un sous-projet portant sur les apprenants de français en Suisse romande (germanophones et italophones), populations pour lesquelles le français jouit d'un statut particulier: le français étant en effet une langue nationale et donc pas complètement " étrangère », les apprenants semblent avoir pour objectif d'acquérir une langue orale telle qu'elle est pratiquée dans la vie de tous les jours, plutôt que viser une norme idéalisée. Le schwa constitue donc un bon indicateur de leur progression dans l'apprentissage de la compétence sociolinguistique (voir également Paternostro et al., 2017).

Dans cette première étude, nous avons examiné le rôle de trois variables (tâche - lecture vs conversation -, type de mots - monosyllabes vs polysyllabes avec un schwa en syllabe initiale vs polysyllabes avec un schwa en syllabe interne - et séjour - séjour d'un ou deux semestres en milieu francophone vs pas de séjour) sur le maintien/chute du schwa dans les productions des apprenants, ainsi que la manière dont ces différents facteurs se combinent.

\subsection{Méthode}

\subsubsection{Participants}

Les données de neuf participants suisses alémaniques avancés de FLE (niveau C1 du Cadre Européen Commun de Référence pour les langues, Conseil de l'Europe, 2001), participant au projet IPFC-Suisse, ont été utilisées dans cette étude. Ils avaient entre 20 et 45 ans et étaient tous originaires de la région zurichoise. Au moment de la collecte des données, ils étudiaient le français au Romanisches Seminar de l'Université de Zurich, en deuxième année, et suivaient environ 15 heures hebdomadaires de cours en français. Cinq d'entre eux avaient effectué auparavant un séjour prolongé (un ou deux semestres) en milieu francophone ${ }^{4}$.

\subsubsection{Matériel et procédure}

Deux des six tâches du protocole IPFC, à savoir le texte lu et la conversation guidée, ont été utilisées dans cette étude. Les participants disposaient de cinq minutes pour prendre connaissance du texte et le préparer avant de le lire à voix haute dans une cabine insonorisée. Pour la conversation guidée, la passation se déroulait dans une pièce calme. L'enquêteur posait des questions fermées et ouvertes à l'apprenant. La transcription a été effectuée sous Praat par un locuteur natif et vérifiée par un deuxième natif. La tire de transcription a ensuite été dupliquée et le codage schwa y a été inséré. Les mots comportant un schwa, qu'il soit réalisé ou non, ont ensuite été codés sur une base perceptive par un locuteur natif ${ }^{5}$. 


\subsubsection{Analyse des données}

Au total, 2'495 occurrences de mots comportant un schwa potentiel ont ainsi été codées (568 pour la lecture et 1'927 pour la conversation). Les données ont ensuite été analysées par le biais de l'interface IPFC de Dolmen. Nous avons exclu de l'analyse les contextes dans lesquels le schwa se maintient généralement toujours, à savoir lorsqu'il est suivi immédiatement par une pause ou une hésitation - indiqué 10 et 20 dans le champ 4 du codage - ou lorsqu'il est réalisé avec une hésitation - indiqué 3 dans le champ 5. Cela représentait 206 occurrences, sur les 2'495 codées. Au final, 2'289 occurrences - 556 pour la lecture et 1'733 pour la conversation - ont été analysées. Des analyses statistiques (modèles mixtes) ont ensuite été réalisées par le biais du logiciel $R$ Studio $^{6}$.

\subsection{Résultats et discussion}

Nous avons examiné le rôle de trois variables sur le maintien/chute du schwa, à savoir le fait d'avoir effectué un séjour de longue durée en milieu francophone (avec vs sans séjour), la tâche (lecture vs conversation) et le type de mots (monosyllabes, syllabe initiale et syllabe interne de polysyllabes). Nous présentons tout d'abord les effets de chaque variable sur le comportement du schwa chez les apprenants alémaniques, avant de nous intéresser à la manière dont elles se combinent.

\subsubsection{Séjour}

Si les études précédentes portant sur les apprenants anglophones (Regan et al., 2009, Thomas, 2001 et Uritescu et al., 2002 et 2004) ont montré qu'un séjour prolongé dans une région francophone a un effet significatif sur le comportement du schwa en permettant généralement aux apprenants d'adopter des usages s'approchant de ceux des natifs, de manière assez surprenante, nos données ne révèlent pas d'effet global de séjour $(F(1,7)=$ 2.48, n. s.). Nous verrons toutefois par la suite que, si le séjour n'agit pas toutes tâches et tous types de mots confondus, il a tout de même un effet significatif lorsqu'il se combine avec l'une ou l'autre des deux autres variables.

\subsubsection{Tâche}

Concernant la tâche, selon les données issues d'études portant sur des natifs, le schwa est d'une manière générale moins enclin à chuter dans une tâche de lecture qu'en conversation (voir par exemple Hansen, 1994, Lucci, 1983, Lyche, 2016). Pour les apprenants, tant Thomas (2001) qu'Uritescu et al. (2002) ont montré que les anglophones canadiens semblent avoir intégré que le comportement du schwa varie en fonction du type de discours puisque le schwa était davantage maintenu en lecture qu'en conversation. C'est aussi le cas des apprenants alémaniques puisque, pour l'ensemble des apprenants (avec et sans séjour), tous types de mots confondus, nos données révèlent également un effet de tâche ( $\mathrm{F}(1$, $\left.\left.2^{\prime} 283\right)=166.62, \mathrm{p}<0.001\right)$, avec un pourcentage de chute nettement plus élevé en conversation, avec $36.82 \%$ (638/1'733 occurrences), qu'en lecture, avec 8.63\% (48/556 occurrences).

\subsubsection{Type de mots}

Un autre facteur qui semble avoir un impact important sur le maintien/chute du schwa est le type de mots. Les données portant sur des locuteurs natifs (Hansen, 1994, Lucci, 1983, Lyche, 2016) montrent que, lorsqu'il est précédé d'une seule consonne (cf. loi des trois consonnes, Grammont, 1914), en syllabe interne de polysyllabe (ex. "souvenir »), sa chute est quasiment systématique, alors que son comportement est beaucoup plus variable dans 
les monosyllabes (ex. «le ») ainsi qu'en syllabe initiale de polysyllabe (ex. « la semaine »). A nouveau, le comportement des apprenants alémaniques - comme c'était également le cas des apprenants anglophones dans les études d'Uritescu et al. (2002 et 2004) - s'approche de celui des natifs. Pour l'ensemble des apprenants (avec et sans séjour), toutes tâches confondues, nos données révèlent en effet un effet du type de mots $\left(\mathrm{F}\left(2,2{ }^{\prime 2} 27\right)=204.11\right.$, $\mathrm{p}<0.001$ ), avec un taux de chute en syllabe interne de polysyllabes significativement plus élevé $(77.90 \%$, soit 208/267 occurrences) que pour les deux autres catégories (monosyllabes : $24.69 \%$, soit 443/1'794 occurrences, $\mathrm{p}<0.001$; syllabe initiale de polysyllabe : $15.35 \%$, soit $35 / 228$ occurrences, $\mathrm{p}<0.001$ ). On peut également relever que le taux de chute est significativement plus élevé pour les monosyllabes qu'en syllabe initiale de polysyllabe $(\mathrm{p}<0.01)$.

\subsubsection{Tâche et type de mots}

Nos résultats montrent également une interaction entre la tâche et le type de mots (F (2, $\left.\left.2^{\prime} 272\right)=4.15, \mathrm{p}<0.05\right)$, ce qui signifie que, chez les apprenants, l'impact de la tâche n'est pas le même pour tous les types de mots. En effet, comme le montre la Figure 2 ci-dessous, l'effet de tâche observé en 3.2.2 - avec un taux de chute beaucoup plus élevé en conversation qu'en lecture - est en fait majoritairement dû aux monosyllabes, avec une très forte différence $(27.30 \%, \mathrm{p}<0.001)$ entre la lecture, avec $4.09 \%$ (18/440 occurrences), et la conversation, avec $31.39 \%$ (425/1'354 occurrences).

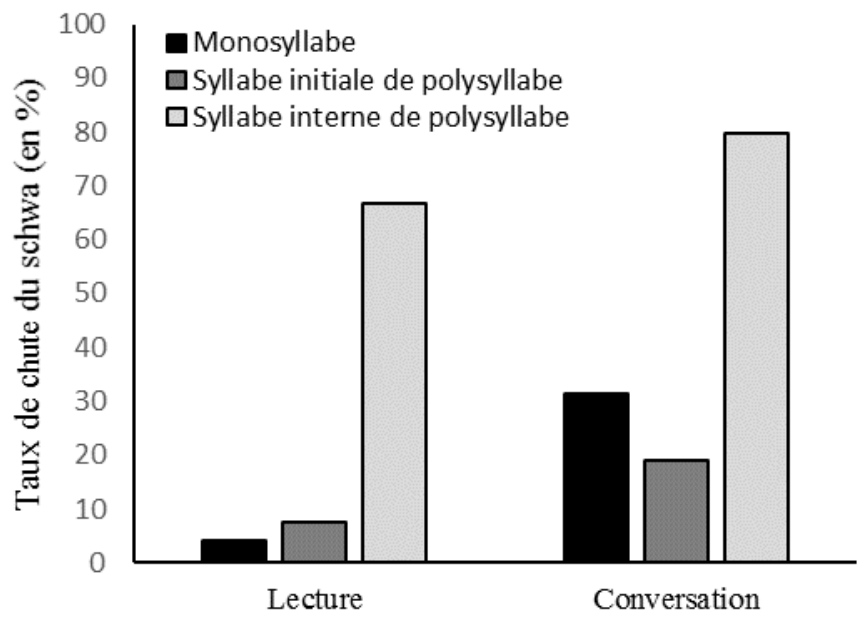

Fig. 2. Taux de chute du schwa (en \%) en fonction de la tâche effectuée (lecture et conversation) et du type de mots (monosyllabes, syllabe initiale et syllabe interne de polysyllabe) chez les apprenants alémaniques.

Pour les deux autres catégories - qui ont un nombre d'occurrences beaucoup plus faible que pour les monosyllabes -, même si le taux de chute est également légèrement plus élevé en conversation qu'en lecture, la différence n'est toutefois pas significative. En syllabe initiale, on passe de $7.50 \%$ (6/80 occurrences) à $19.59 \%$ (29/148 occurrences, n. s.) et, en syllabe interne, on passe de $66.67 \%$ (24/36 occurrences) à $79.65 \%$ (184/231 occurrences, n. s.).

En s'appuyant sur les données issues de 13 points d'enquête du projet PFC (143 locuteurs de la partie nord de la France), Lyche (2016) fournit des taux de chute pour les 
monosyllabes et la syllabe initiale de polysyllabe pouvant servir de points de comparaison avec nos données. Si les taux de chute qu'elle indique sont également plus bas en lecture (monosyllabes : $8.62 \%$, syllabe initiale : $1.47 \%$ ) qu'en conversation (monosyllabes : $65.39 \%$, syllabe initiale : $65.82 \%$ ), on retiendra surtout que, pour les deux contextes, les taux de chute en conversation chez les apprenants alémaniques sont très nettement inférieurs à ceux des natifs, ce qui était également le cas des apprenants anglophones des études d'Uritescu et al. (2002 et 2004).

\subsubsection{Tâche et séjour}

Comme nous l'avons vu précédemment - et ce de manière à première vue surprenante -, le séjour ne semble pas, à lui seul, avoir un impact sur le comportement du schwa. Toutefois, nos résultats montrent qu'il interagit de manière significative avec la tâche $(\mathrm{F}(1,2$ '281) $=$ $5.00, \mathrm{p}<0.05)$. Comme le montre la Figure 3 ci-dessous, le fait d'avoir effectué un séjour en milieu francophone n'influence pas de manière identique le comportement du schwa dans les deux tâches. En effet, si le séjour n'a pas d'effet en lecture - avec, pour les apprenants alémaniques sans séjour, un taux de chute de $8.50 \%$ (21/247 occurrences) contre $8.74 \%$ (27/309 occurrences) pour ceux ayant effectué un séjour (n. s.) -, il semble avoir un impact important en conversation, avec un taux de chute du schwa significativement plus élevé chez les apprenants ayant effectué un séjour (40.19\%, 428/1'065 occurrences) que chez ceux sans séjour $(31.43 \%, 210 / 668$ occurrences, $\mathrm{p}<0.05)$. On peut relever ici que c'est justement en conversation que le comportement des apprenants se distinguait le plus de celui des natifs, avec des taux de chute très largement inférieurs. Le séjour semble donc permettre aux apprenants de s'approcher davantage des usages des natifs.

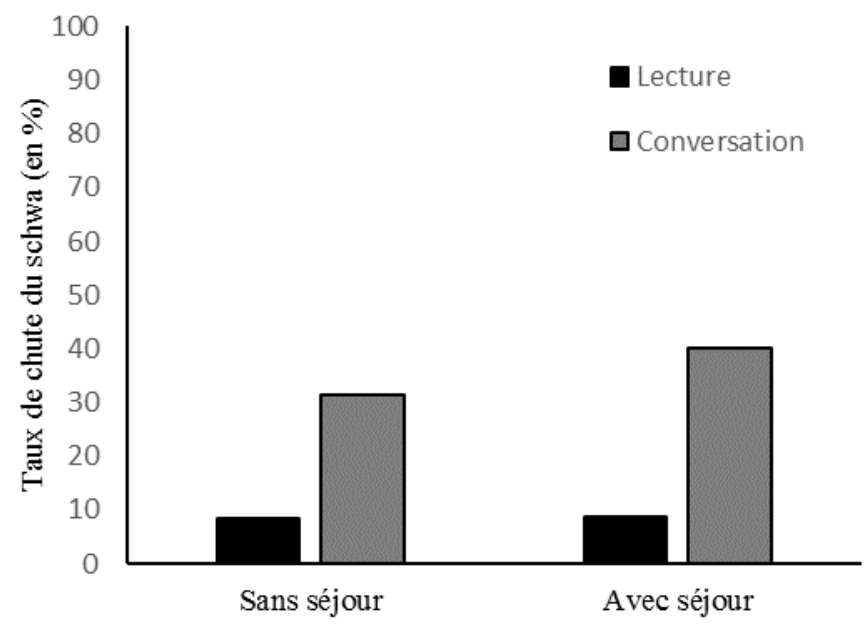

Fig. 3. Taux de chute du schwa (en \%) en fonction du séjour (sans et avec) et de la tâche effectuée (lecture et conversation) chez les apprenants alémaniques.

\subsubsection{Type de mots et séjour}

Nos données révèlent également une interaction significative entre le séjour et le type de mots $(\mathrm{F}(2,2 \nmid 275)=3.11, \mathrm{p}<0.05)$. La Figure 4, ci-dessous, montre que le fait d'avoir effectué un séjour n'a pas le même effet sur les trois types de mots, améliorant le taux de chute du schwa de manière significative uniquement pour les monosyllabes, avec pour les apprenants sans séjour, un taux de chute de $17.68 \%$ (122/690 occurrences), contre $29.08 \%$ $(321 / 1$ '104 occurrences) pour ceux qui ont effectué un séjour $(\mathrm{p}<0.05)$. Le séjour n'a en revanche aucune influence sur le comportement du schwa de polysyllabe, qu'il soit en 
syllabe interne (taux de chute pour les apprenants sans séjour : 76.03\%, 92/121 occurrences vs $79.45 \%, 116 / 146$ occurrences pour ceux avec séjour, n. s.) ou en syllabe initiale (sans séjour : $16.35 \%, 17 / 104$ occurrences vs avec séjour : 14.52\%, 18/124 occurrences, n. s.).

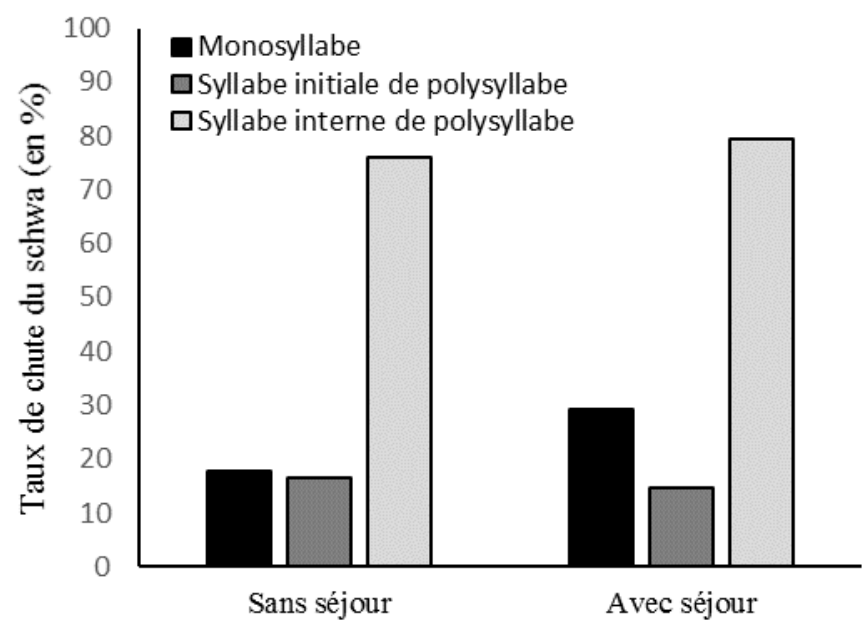

Fig. 4. Taux de chute du schwa (en \%) en fonction du séjour (sans et avec) et du type de mots (monosyllabe, syllabe initiale et syllabe interne de polysyllabe) chez les apprenants alémaniques.

Ainsi, si le séjour semble agir davantage sur le comportement des apprenants en conversation, en faisant augmenter le taux de chute du schwa, ce résultat indique que c'est sur les monosyllabes que se concentre cet effet du séjour, ce qui ressort également des données d'Uritescu et al. (2002). Le codage du schwa que nous avons proposé permettant d'examiner de manière plus détaillée le comportement du schwa en fonction du contexte segmental qui le précède - indiqué par le champ 3 du codage -, nous proposons d'affiner ce résultat en examinant l'effet du contexte segmental pour ce contexte précis, à savoir les monosyllabes produits en conversation.

\subsubsection{Environnement segmental gauche pour les monosyllabes produits en conversation}

Trois contextes ont été examinés : a) lorsque le schwa est précédé de deux consonnes $(\mathrm{C}(\#) \mathrm{C} ə)$; b) lorsque le schwa est précédé d'une seule consonne $(\mathrm{V}(\#) \mathrm{C} ə)$ et c) lorsque le schwa est précédé d'une seule consonne située en début de groupe intonatif ou elle-même précédée d'une pause ou d'une hésitation (Cə). Nos données montrent une interaction entre le séjour et le contexte gauche $\left(\mathrm{F}\left(2,1{ }^{\prime} 382\right)=5.54, \mathrm{p}<0.01\right)$, ce qui signifie que le séjour n'agit pas de la même manière selon le contexte segmental qui précède.

La Figure 5, ci-dessous, montre que le taux de chute du schwa dans les monosyllabes produits en conversation est significativement plus élevé chez les apprenants ayant effectué un séjour dans les deux contextes où le schwa est précédé d'une seule consonne, que celleci soit en début de groupe intonatif, précédée d'une pause, d'une hésitation (Cə) ou d'une voyelle $(\mathrm{V}(\#) \mathrm{C} ə)$. En effet, pour le contexte $\mathrm{C}$, le taux de chute double entre les apprenants sans séjour $(21.43 \%, 24 / 112$ occurrences) et ceux avec séjour $(42.42 \%, 70 / 165$ occurrences, $\mathrm{p}<0.001$ ) et l'augmentation est presque aussi élevée pour le contexte $\mathrm{V}(\#) \mathrm{C} \partial$, avec un taux de chute de $22.22 \%$ (62/279 occurrences) pour les apprenants sans séjour, contre $39.17 \%$ $(199 / 508$ occurrences) pour ceux avec séjour $(\mathrm{p}<0.001)$. En revanche, lorsque le schwa est précédé de deux consonnes $(\mathrm{C}(\#) \mathrm{C})$, le taux de chute ne diffère pas, avec $26.47 \%(27 / 102$ 
occurrences) pour les apprenants sans séjour contre $22.16 \%$ (41/185 occurrences) pour ceux avec séjour (n. s.).

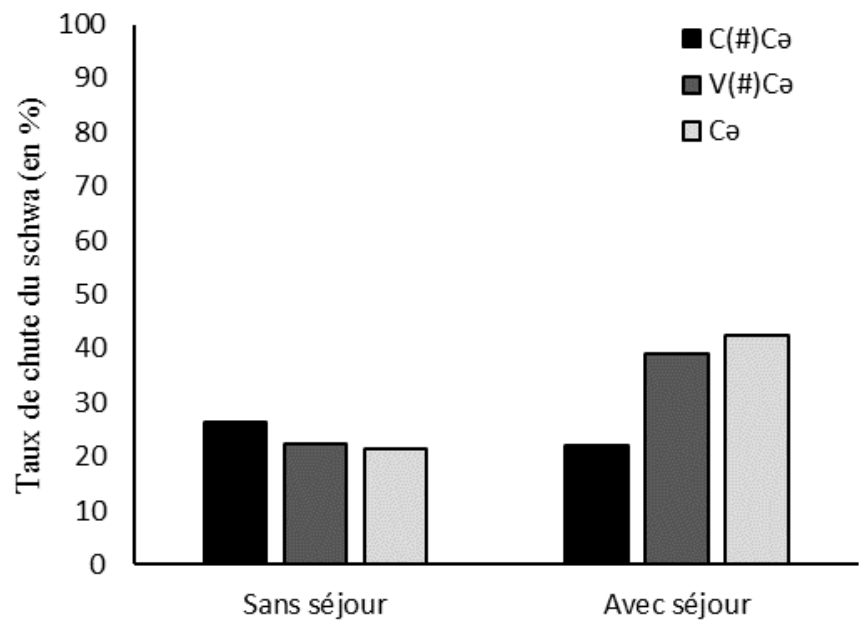

Fig. 5. Taux de chute du schwa (en \%) pour les monosyllabes produits en conversation, en fonction de l'environnement segmental précédant $(\mathrm{C}(\#) \mathrm{C} ə, \mathrm{~V}(\#) \mathrm{C}$ ə et $\mathrm{C} ə)$ et du séjour (sans et avec) chez les apprenants alémaniques.

Ces résultats montrent que, pour le contexte où le séjour a le plus d'effet, à savoir les monosyllabes produits en conversation, il agit également en permettant aux apprenants de différencier les occurrences de schwa en fonction du contexte qui précède, ce qu'ils ne semblaient pas faire auparavant. On observe par conséquent que la loi des trois consonnes est davantage appliquée après un séjour de longue durée en milieu francophone, en tout cas pour les monosyllabes produits en conversation, puisque, chez les apprenants avec séjour, le schwa chute davantage dans les deux contextes où il n'est précédé que d'une seule voyelle $(\mathrm{V}(\#) \mathrm{C}$ ə et $\mathrm{C}$ ə) que lorsqu'il est précédé de deux consonnes $(\mathrm{C}(\#) \mathrm{C} ə)$ - et ce de manière significative, $\mathrm{p}<0.001$ les deux fois) -, ce qui n'est pas le cas pour les apprenants sans séjour, où les trois contextes ne se différencient pas les uns des autres (n. s.).

\section{Conclusion}

Les résultats préliminaires présentés ci-dessus montrent que l'approche par le biais d'un codage combinant champs descriptifs et perceptifs permet d'effectuer une analyse fine du comportement du schwa chez les apprenants de FLE. Ainsi, si les études précédentes (Regan et al., 2009, Thomas, 2001, Uritescu et al., 2002 et 2004) ont montré qu'avec une expérience en immersion, les usages des apprenants tendent à se rapprocher de ceux des natifs, nos données affinent ces résultats : le séjour agit sur le comportement du schwa produit par des apprenants alémaniques avancés de manière très ciblée, à savoir uniquement dans les monosyllabes produits en conversation et précédés d'une seule consonne, ellemême précédée d'une voyelle, d'une pause ou d'une hésitation. Même si le taux de chute pour ce contexte reste encore largement inférieur à celui observé chez les natifs, le séjour permet donc aux apprenants alémaniques d'améliorer leur compétence sociolinguistique en situation de conversation, mais uniquement pour les monosyllabes. Ils sont en outre en mesure d'appliquer de manière adéquate, pour ce contexte, la loi des trois consonnes, qui est enseignée dans les manuels de prononciation. En revanche, si l'on examine l'autre contexte variable chez les natifs, la syllabe initiale de polysyllabe, outre le fait que le taux de chute y est largement inférieur à celui observé chez les natifs, on constate que le séjour n'a pas d'impact. Paternostro et al. (2017) ayant montré une gradation dans l'appropriation 
de deux contextes variables - la chute du «ne » de négation semblant être acquise avant celle du schwa dans les structures «je + consonne » (ex. « je peux») -, on peut faire l'hypothèse que la durée du séjour - qui était d'un à deux semestres pour les apprenants de notre étude - n'est peut-être pas suffisante pour agir sur le schwa en syllabe initiale de polysyllabes.

Au niveau méthodologique, ces premiers résultats permettent de valider, pour le schwa également, l'approche par codage développée dans le projet IPFC. Appliquée à grande échelle sur des populations diverses en termes de niveau aussi bien que de L1, elle devrait permettre de dresser un panorama de l'apprentissage du schwa par les apprenants de FLE. $\mathrm{Au}$ niveau didactique, ce travail devrait aboutir, à terme, à un renouvellement des ressources pédagogiques afin de ne pas laisser l'appropriation du schwa à la portée des seuls apprenants en mesure d'effectuer un séjour en immersion - de surcroît d'une durée supérieure à deux semestres - mais de la travailler également de manière efficace en classe.

\section{Références}

Abry, D. \& Chalaron, M.-L. (2011). Les 500 exercices de phonétique avec corrigés, niveau B1/B2. Paris : Hachette.

Andreassen, H. N. \& Lyche, C. (2016). Le schwa dans la parole conversationnelle d'apprenants et l'impact des facteurs phonologiques: un début d'analyse. Rencontres Floral- PFC 2016 Journées IPFC, Paris, 21-22 novembre 2016, http://hdl.handle.net/10037/10220.

Boersma, P. \& Weenink, D. (2015). Praat: doing phonetics by computer, version 5.4 .04 retrieved from http://www.praat.org.

Boubnova, G. \& Ratnikova, E. (2016). La norme des locuteurs non natifs et son évaluation en L2 : le [ə] instable dans la production orale des russophones (niveau de référence B1-B2). Rencontres Floral- PFC 2016 - Journées IPFC, Paris, 21-22 novembre 2016.

Charliac, L. \& Motron, A.-C. (2014). Phonétique progressive du français, avec 600 activités, niveau intermédiaire ( ${ }^{\text {ème }}$ édition). Paris : CLE International.

Conseil de l'Europe (2001). Cadre européen commun de référence pour les langues : apprendre, enseigner, évaluer. Paris : Didier.

Delais-Roussarie, E. (2009). Conventions CHAT de Transcription des données. Document interne, BDD Interlangue.

Derwing, T. M. \& Munro, M. J. (2015). Pronunciation Fundamentals: Evidence-based Perspectives for L2 teaching and research. Amsterdam: John Benjamins.

Detey, S. (2012). Coding an L2 phonological corpus : from perceptual assessment to non-native speech models - an illustration with French nasal vowels. In: Tono, Y., Kawaguchi, Y., Minegishi, M. (eds). Developmental and Crosslinguistic Perspectives in Learner Corpus Research. Amsterdam and Philadelphia: John Benjamins, 229-250.

Detey, S. (2014). Vers une évaluation par codage perceptive sur corpus de la production des liquides françaises $/ \mathrm{R} /$ et $/ 1 /$ des apprenants japonais en singleton et en groupe consonantique. Flambeau, 40, 1-17.

Detey, S. \& Kawaguchi, Y. (2008). Interphonologie du Français Contemporain (IPFC) : récolte automatisée des données et apprenants japonais. Journées PFC 2008: variation, interfaces, cognition, Paris, 11-13 décembre 2008.

Detey, S. \& Racine, I. (2017). Toward a perceptually assessed corpus of non-native French : the InterPhonology of Contemporary French (IPFC) project illustrated with a longitudinal study of Japanese learners' /b-v/ productions. In: Trouvain, J., Zimmerer, F., Möbius, B., Gósy, M. \& Bonneau, A. (eds), Investigating segmental, prosodic and fluency features in spoken learner corpora, Special issue of International Journal of Learner Corpus Research, 3, 2, 223-249. 
Detey, S., Racine, I., Eychenne, J. \& Kawaguchi, Y. (2014). Corpus-based L2 phonological data and semi-automatic perceptual analysis: the case of nasal vowels produced by beginner Japanese learners of French. Proceedings of Interspeech 2014, 539-544.

Detey, S., Racine, I., Kawaguchi, Y. \& Eychenne, J. (2016a). Le domaine segmental (vocalique et consonantique). Dans : Detey, S., Racine, I., Kawaguchi, Y., Eychenne, J. (éds), La prononciation du français dans le monde : du natif à l'apprenant. Paris : CLE International, 236-237.

Detey, S., Racine, I., Kawaguchi, Y. \& Zay, F. (2016b). Variation among non-native speakers: The InterPhonology of Contemporary French. In: Detey, S., Durand, J., Laks, B. \& Lyche, C. (eds), Varieties of Spoken French. Oxford: Oxford University Press, 491-502.

Durand, J. (2009). On the scope of linguistics: data, intuitions, corpora. In: Kawaguchi, Y., Minegishi, M., Durand, J. (eds), Corpus Analysis and Variation in Linguistics. Amsterdam: John Benjamins:, 25-52.

Durand, J., Laks, B. \& Lyche, C. (éds) (2009). Phonologie, variation et accents du français. Paris : Hermès.

Durand, J. \& Lyche, C. (2003). Le projet Phonologie du français contemporain (PFC) et sa méthodologie. Dans : Delais-Roussarie, E., J. Durand (éds), Corpus et variation en phonologie du français : méthodes et analyse. Toulouse : Presses universitaires du Mirail, 213-276.

Eychenne, J. \& Paternostro, R. (2016). Analyzing transcribed speech with Dolmen. In: Detey, S., Durand J., Laks, B. \& Lyche, C. (eds), Varieties of Spoken French. Oxford: Oxford University Press, D35-D52.

Grammont, M. (1914). Traité pratique de prononciation française. Paris : Delagrave.

Hansen, A. B. (1994). Etude du E caduc - stabilisation en cours et variations lexicales. Journal of French Language Studies, 4, 25-54.

Howard, M., Mougeon, R. \& Dewaele, J.-M. (2013). Sociolinguistics and second language acquisition. In: Bayley, R., Cameron, R., Lucas, C. (eds), The Oxford Handbook of Sociolinguistics. Oxford : Oxford University Press, 340-359.

Katanaeva-Morozova, O. (2008). Le phénomène phonologique du «e» muet en français. Essai non publié pour l'obtention du DEFLE, Université de Genève.

Laks, B. (2015). Histoire de la liaison, cours donné dans le cadre de l'école d'été PFC: «PFC: phonologie, corpus et méthodes », Université de Vienne, 13-18 juillet 2015.

Lucci, V. (1983). Prosodie, phonologie et variation en français contemporain. Dans : Walter, H. (éd.), Langue française, 60 - Phonologie des usages du français, 73-84.

Lyche, C. (2016). Approaching variation in PFC: the schwa level. In: Detey, S., Durand J., Laks, B. \& Lyche, C. (eds), Varieties of Spoken French. Oxford: Oxford University Press, 352-262.

Nouveau, D. (2012). Limites perceptives de l'e caduc chez les apprenants néerlandophones. Revue canadienne de linguistique appliquée, 15, 1, 60-78.

Nouveau, D. \& Detey, S. (2007). Enseignement/apprentissage du schwa et apprenants néerlandais : des données de la base PFC à l'espace-ressource en ligne du projet PFC-EF. Bulletin PFC, 7, 87106.

Mac Whinney, B. (2015). The CHILDES project: Tools for Analyzing Talk, http://citeseerx.ist.psu.edu/viewdoc/download?doi=10.1.1.693.5831\&rep=rep1\&type=pdf. Consulté le 10 novembre 2017.

Martins, C. \& Mabilat, J.-J. (2004). Sons et intonation : exercices de prononciation. Paris : Didier.

Mougeon, R., Nadasdi, T. \& Rehner, K. (2002). Etat de la recherche sur l'appropriation de la variation par les apprenants avancés du FL2 ou FLE. Acquisition et interaction en langue étrangère, 17, 7-50. Consulté le 20 octobre 2017 sur : http://aile.revues.org/847. 
Paternostro, R., Didelot, M. \& Racine, I. (2017). Quelques traits stylistiques chez les apprenants italophones de FLE. Repères DoRiF, http://www.dorif.it/ezine/show issue.php?iss id=23 (DoRiF Università, Rome).

Racine, I. (2008). Les effets de l'effacement du schwa sur la production et la perception de la parole en français. Thèse de doctorat non publiée, Université de Genève. Consulté le 15 décembre 2017, sur : https://archive-ouverte.unige.ch/unige:602.

Racine, I. (2012). Spanish learners' productions of French close rounded vowels: a corpus-based perceptual study. In: Tono, Y., Kawaguchi, Y., Minegishi, M. (eds), Developmental and Crosslinguistic Perspectives in Learner Corpus Research. Amsterdam: John Benjamins, 205-228.

Racine, I., Detey, S., Zay, F. \& Kawaguchi, Y. (2012). Des atouts d'un corpus multitâches pour l'étude de la phonologie en L2: l'exemple du projet "Interphonologie du français contemporain » (IPFC). Dans : Kamber, A., Skupiens, C. (éds). Recherches récentes en FLE. Berne : Peter Lang, 1-19.

Racine, I. \& Detey, S. (sous presse). Production of French close rounded vowels by Spanish learners : a corpus-based study. In: Gibson, M. \& Gil Fernández, J. (eds), Current Studies in Romance Phonetics and Phonology. Oxford: Oxford University Press.

Racine, I., Zay, F., Detey, S. \& Kawaguchi, Y. (2011). De la transcription de corpus à l'analyse interphonologique: enjeux méthodologiques en FLE. Dans: Col, G., Osu, S. (éds), Travaux Linguistiques du CerLiCO 24, Rennes : PUR, 13-30.

Regan, V., Howard, M. \& Lemée, I. (2009). The Acquisition of Sociolinguistic Competence in a Study Abread Context. Bristol/Buffalo/Toronto: Multilingual Matters.

Stridfeldt, M. (2005). La perception du français oral par des apprenants suédois. Thèse de doctorat, Umeå Universitet.

Thomas, A. (2001). Schwa au niveau avancé du français langue seconde. Revue canadienne de linguistique appliquée, 4, 1-2, 103-112.

Uritescu, D., Mougeon, R. \& Handouleh, Y. (2002). Le comportement du schwa dans le français parlé par les élèves des programmes d'immersion française. Dans: Tatilon, C. \& Baudot, A. (éds), La Linguistique Fonctionnelle au Tournant du Siècle. Actes du 24e Colloque International de Linguistique Fonctionnelle. Toronto : GREF, 335-346.

Uritescu, D., Mougeon, R., Rehner, K. \& Nadasdi, T. (2004). Acquisition of the internal and external constraints of variable schwa deletion by French immersion students. International Review of Applied Linguistics in Language Teaching, 42, 349-364.

Nous tenons à remercier chaleureusement Inka Wissner, Stefan Schmid ainsi que tous les apprenants alémaniques qui ont participé à cette recherche, Marion Didelot et Roberto Paternostro, pour leur précieux travail de transcription des données, ainsi que Chantal Lyche et Jeff Tennant, pour leur collaboration à l'élaboration du codage schwa. Le projet IPFC-Suisse bénéficie du soutien du Fonds national suisse de la recherche scientifique (no 169707).

\footnotetext{
${ }^{1}$ Notre description se limite ici à des usages qui ne sont pas ceux des variétés méridionales de français, la dichotomie entre le nord et le sud de la France concernant le comportement du schwa ayant largement été documentée, comme le souligne Lyche (2016).

${ }^{2}$ Où $\mathrm{V}=$ voyelle, \# frontière entre deux mots et $\mathrm{C}=$ consonne. La parenthèse entourant le \# permet d'inclure aussi bien les contextes dans lesquels il y a une frontière de mot entre $\mathrm{V}$ et $\mathrm{C}$ (ex. «la semaine ») que ceux où il n'y en a pas (ex. " casserole »), indication qui peut être extraite du champ 2 et qui serait donc redondante ici.

${ }^{3}$ Le schwa du «le» n'est pas codé, la présence d'une double transcription, indiquée entre parenthèses, signalant que les transcripteurs ne sont pas sûrs de la présence du «le », qui n'est pas audible distinctement. La séquence a pu être produite sans déterminant («je parle suisse allemand »).
} 
${ }^{4}$ Ces séjours ont été effectués soit en Suisse romande, soit en France septentrionale. En raison de la dichotomie bien documentée entre le nord et le sud de la France concernant le comportement du schwa (Lyche, 2016), nous avons exclu de notre corpus d'étude deux apprenants ayant séjourné pour l'un, à Marseille et, pour l'autre, à Bordeaux.

${ }^{5}$ A noter ici que le codage devra encore être vérifié par un deuxième codeur. Toutefois, l'étape de codage étant chronophage, pour des raisons de temps et de ressources, cette étape n'a pas encore pu être réalisée.

${ }^{6}$ Pour des raisons de clarté, les résultats et les graphes sont présentés en pourcentages, bien que toutes les analyses aient été effectuées à partir des données brutes. 